\section{Um modelo para avaliação rápida da situação nutricional e de saúde de crianças e mães no "dia nacional de vacinação".}

\section{A model for quick evaluation of the nutritional and health situation of mothers and children on the "national vaccination day"}

Malaquias Batista Filho 1

Luiz Oscar Cardoso Ferreira 2

1 Departamento de Nutrição da Universidade Federal de Pernambuco; Instituto Materno Infantil de Pernambuco, IMIP. Departamento de Nutrição, Centro de Ciências da Saúde Universidade Federal de Pernambuco, Campus Universitário, Cidade Universitária, Recife, Pernambuco, Brasil. CEP 50.670 901

2 Departamento de Medicina Social, Faculdade de Ciências Médicas da Universidade de Pernambuco

\section{Abstract}

Objectives: to discuss the experience, conception and development of a simplified model to evaluate the nutritional status and correlated health situation of mothers and children using the "national vaccination day" as strategy, when $90 \%$ of children and $80 \%$ of mothers appear at the vaccination center.

Methods: five health units of vaccination were $o b-$ served in a town from the coastal area (Zona da Mata) of Pernambuco. The sample size was estimated considering a prevalence error from $3 \%$ to $5 \%$ in relation to the basic variables of interest, such as, prevalence of malnutrition, anemia, diarrhea, acute respiratory infection, breastfee-ding, monitoring of growth and development and vaccine situation, and four aspects from mothers: body mass index, prevalence of anemia, antenatal care and educatio-nal level.

Results: the study involved a sample of 579 children and 417 mothers. Among the mothers, 80,3\% were literate. 18,0\% of the children had had acute respiratory infection in the last week, while only 7,4\% of those who were from six to 12 months had an incomplete vaccine situation in relation to Sabin and DPT. $37,8 \%$ were anemic and $11 \%$ showed stature deficit. The mean and the median of exclusive breastfeeding were 11,5 and 9,0 days, respectively. We observed twice as many obese women as thin women $(10,6 \% ; 4,9 \%$ respectively), and $16,2 \%$ were anemic.

Conclusions: the results were similar to the expected values, compared with previous epidemiological studies that took place in Pernambuco state, which showed the validity of the simplified model in Brazil and others countries that use a "national vaccination day" as a strategy. Key words Nutrition assessment, Maternal and child health

\section{Resumo}

Objetivos: relatar a experiência com a concepção $e$ desenvolvimento de um modelo simplificado de avaliação do estado nutricional e situações correlatas de saúde materno-infantil, aproveitando o "dia nacional de vacinação" como estratégia, quando $90 \%$ das crianças e $80 \%$ das mães comparecem aos postos de atendimento

Métodos: cinco postos de vacinação de uma cidade da Zona da Mata de Pernambuco foram pesquisados. A amostra, dimensionada para um erro de prevalência de $3 \%$ a $5 \%$ em relação às variáveis básicas, foi representada pela prevalência de desnutrição, anemia, diarréia, infecção respiratória aguda (IRA), amamentação/desmame, vigilância de crescimento e desenvolvimento e situação vacinal, além de quatro aspectos referentes às mães: índice de massa corporal, prevalência de anemia, cuidados pré-natais e nível de educação.

Resultados: foram observadas 579 crianças e 417 mães. 80,3\% das mães eram alfabetizadas. Entre as crianças 18,0\% apresentaram IRA nos últimos 7 dias. Nas de seis a 12 meses apenas 7,4\% não tinham completado o esquema vacinal com a Sabin e a DPT. 37,8\% eram anêmicas e $11 \%$ tinham déficit estatural. A média e a mediana da amamentação exclusiva foram de 11,5 e 9,0 dias, respectivamente. Constatou-se que há duas vezes mais mulheres obesas do que magras (10,6\% e 4,9\%), e que $16,2 \%$ eram anêmicas.

Conclusões: os resultados concordam com os valores esperados, a partir de estudos prévios em Pernambuco, demonstrando a validade da aplicação do modelo no Brasil e outros países que utilizam esta estratégia. Palavras-chave Avaliação nutricional, Saúde materno-infantil 


\section{Introdução}

Embora coexistindo com outros problemas epidemiológicos desde os primórdios da humanidade, somente na segunda metade do século atual as doenças carenciais e, mais recentemente, o trinômio obesidade/diabetes/doenças cardiovasculares de caráter degenerativo, passaram a ocupar um espaço de destaque em nível de estudos epidemiológicos e no cenário das políticas e programas de saúde. Esta valorização se formalizou, historicamente, nas reuniões de Alma-Ata em 1978, ${ }^{1}$ Nova Iorque em 1990, 2 e Roma em 1992.3

Entre as 15 grandes prioridades de saúde para o ano 2000 , seis estavam representadas por problemas alimentares/nutricionais, implicando no compromisso de promover a segurança alimentar em nível de família, o aleitamento materno exclusivo nos quatro ou seis primeiros meses de vida, a redução do baixo peso ao nascer, da desnutrição energético-protéica moderada e grave em crianças, o controle epidemiológico das deficiências de iodo e de vitamina A e a diminuição, em pelo menos $1 / 3$, na prevalência de anemia em mulheres no ciclo gravídico. ${ }^{2}$ Essas situações interessam, fundamentalmente, ao segmento materno-infantil da população.

Além de seu significado como nosologias específicas, as doenças da nutrição apresentam a singularidade de se associarem a um amplo espectro de enfermidades, principalmente de natureza infecciosa, nos países pobres, ou crônico-degenerativas, nas nações mais desenvolvidas. $\mathrm{Na}$ primeira condição, os problemas carenciais estão associados a $55 \%$ dos óbitos infantis, enquanto na outra polarização correlacionam-se com mais de $40 \%$ das mortes nu-cleadas pelas doenças cardiovasculares. 4

Esta sinopse põe em relevo a importância dos problemas relacionados com a nutrição, no contexto do quadro de saúde. Seu conhecimento, portanto, representa um quesito básico para a definição de políticas globais e específicas.

Um requisito fundamental para a orientação satisfatória das decisões e monitoramento de sua implementação, consiste na disponibilidade de dados confiáveis e atualizados nos diferentes espaços geográficos. É o que se propõe, desde 1974, quando foi concebida a vigilância alimentar e nutricional, 5 consolidada nas reuniões de Alma-Ata, em 1978,1 Nova Iorque em 1990,2 Roma em $1992^{3}$ e, ainda com maior ênfase, nas reuniões periódicas da Rede de Cooperação Técnica sobre Vigilância Alimentar e Nutricional na América Latina e Caribe, realizadas no Chile em 1993 e em 1994, na Venezuela em 1996 e no Recife em 1997.6
Em função desses argumentos e compromissos, um questionamento se estabelece: como obter de forma ágil, segura e a baixo custo, informações que possam oferecer o suporte necessário para a geração, processamento e análise de dados básicos para alimentar os sistemas informativos e, em conseqüência, subsidiar medidas de intervenção e seu seguimento? É uma questão de considerável pertinência, levandose em conta o custo elevado e as dificuldades técnicas dos inquéritos epidemiológicos convencionais para avaliar a situação nutricional e fatores associados.

No caso do Brasil e de outros países que adotam a experiência bem sucedida do "dia nacional de vacinação", dispõe-se de uma oportunidade singular para a proposição e teste de modelos que possam responder a tais desafios. Há uma demanda praticamente universal de mães e crianças aos postos de vacinação, criando condições para que se possa coletar, periodicamente, dados básicos representativos da situação nutricional e outras variáveis de interesse correlato, no campo de interseção da saúde e nutrição.

No projeto experimental aqui proposto e testado, delineia-se uma tentativa inicial de desenho e validação de métodos de avaliação epidemiológica que possam resultar na definição de modelos recomendados para situações específicas, como no caso peculiar dos "dias nacionais de vacinação" .

Nesta perspectiva, objetivou-se propor, testar e reproduzir métodos simplificados de avaliação do estado de nutrição, problemas de saúde e fatores correlatos de reconhecida importância na gênese da situação nutricional de mães e crianças, que possam ser aplicados de forma rápida e com baixo custo. Especificamente, a proposta aproveita o "dia nacional de vacinação" como oportunidade para avaliar aspectos nutricionais de reconhecida relevância em saúde pública, se discrimina a seguir: 1) em relação às crianças menores de cinco anos: a) história de amamentação no primeiro ano de vida, para os menores de 1 ano; b) cobertura vacinal; c) vigilância do crescimento e desenvolvimento; d) cobertura do programa de atendimento às crianças desnutridas; e) história recente de diarréias, nos últimos 15 dias e infecções respiratórias, nos últimos 7 dias; f) determinação dos níveis de hemoglobina; g) estado nutricional por avaliação antropométrica; 2) em relação às mães: a) freqüência ao pré-natal; b) níveis de hemoglobina; c) índice de massa corporal; d) nível de alfabetização. 


\section{Métodos}

O estudo foi realizado na cidade de Ribeirão, Pernambuco, situada a 92 quilômetros do Recife, capital do estado, no Nordeste brasileiro. Trata-se de um município com 39.900 habitantes, dos quais $72 \%$ radicados na área urbana. ${ }^{7} \mathrm{O}$ Índice de Desenvolvimento Humano (IDH) do município é de 0,460 (IDH do Brasil 0,742; de Pernambuco 0,572). Quase dois terços $(62,8 \%)$ dos chefes de família têm renda de até um salário mínimo ( $1 \mathrm{SM} \cong \mathrm{US} \$ 80,00)$, enquanto que a taxa de analfabetismo entre os jovens de 11 a 14 anos de idade é de $40,7 \%$. A taxa de mortalidade infantil estimada para 1998 era de 68 óbitos por mil nascidos vivos.7

A amostra estudada foi constituída de crianças menores de cinco anos e suas mães, atendidas nos locais utilizados para o "dia nacional de vacinação", assumindo-se, em princípio, que a demanda seja satisfatoriamente representativa do universo de crianças e mães da localidade.

O tamanho amostral foi calculado para representar os atributos principais do estudo, seja por conhecimento prévio do valor provável, seja por referência ao limiar endêmico do problema ou fator objetivado. Em outras situações, assumiu-se como referência para o cálculo amostral as recomendações normativas de cobertura ou, em outras palavras, as metas estabelecidas por normas de serviços ou por compromissos programáticos. Calculou-se o tamanho da amostra, admitindo-se uma população de 5.000 menores de seis anos (aproximadamente 12\% da população total) e um erro alfa de $5 \%$. Admitiuse, também, um erro de 3\% a 5\% para cada uma das prevalências estimadas: a) desnutrição em menores de cinco anos: $10 \%$ para valores abaixo de -2 escores $z$ para a relação altura/idade (A/I); b) história do desmame parcial e completo: prevalência de $40 \%$ e $15 \%$, respectivamente, aos seis meses de idade; c) ocorrência de anemia: $40 \%$ em menores de cinco anos e $25 \%$ para mulheres no período reprodutivo; d) vi-gilância do crescimento: $90 \%$ de notificação no "cartão da criança" nos últimos três meses; e) vigilância do desenvolvimento: $90 \%$ de notificação no "cartão da criança" nos últimos três meses; f) história de diarréia: prevalência estimada de $15 \%$ para os últimos 15 dias; g) história de infecções respiratórias: prevalência estimada de $20 \%$ para os últimos 7 dias; h) índice de massa corporal: prevalência estimada de sobrepeso e obesidade de $20 \%$; i) cobertura do pré-natal: $60 \%$.

A Tabela 1 especifica o resultado do tamanho da amostra com os parâmetros utilizados para o seu cálculo.

Tabela 1

Tamanho da amostra para representatividade das diversas variáveis. Ribeirão, Pernambuco, 1998.

\begin{tabular}{lccc}
\hline \multicolumn{1}{c}{ Variáveis } & $\begin{array}{c}\text { Prevalência } \\
\text { \% }\end{array}$ & Erro da prevalência & Tamanho da Amostra \\
\hline Crianças & & $\%$ & $357-207$ \\
Prevalência de desnutrição (A/I)* & 10 & 3 a 4 & $517-343$ \\
Prevalência do desmame parcial & 40 & 4 a 5 & $491-288$ \\
Prevalência do desmame completo & 15 & 3 a 4 & $517-343$ \\
Prevalência de anemia & 40 & 4 a 5 & $357-207$ \\
Vigilância do CD** & 90 & 3 a 4 & $491-288$ \\
Prevalência de diarréia & 15 & 3 a 4 & $357-234$ \\
Prevalência de IRA*** & 20 & 4 a 5 & $357-207$ \\
Vacinação básica & 90 & 3 a 4 & $415-272$ \\
$\quad$ Mães & & 4 a 5 & $357-234$ \\
Prevalência de anemia & 25 & 4 a 5 & $517-343$ \\
Índice de massa corporal & 20 & 4 a 5 & \\
Freqüência ao pré-natal & 60 & & \\
\hline
\end{tabular}

* $\mathrm{A} / \mathrm{I}=$ Altura para idade; $* * \mathrm{CD}=$ Crescimento e desenvolvimento; ** $\mathrm{IRA}=$ Infecção Respiratória Aguda 
O tamanho da amostra foi definido tomando-se como número de eleição o valor mais elevado, ou seja 517 sujeitos. Prevendo-se a ocorrência de perdas e inconsistências, elevou-se a amostra para 550 crianças, a partir das quais seriam identificadas e examinadas as respectivas mães.

Cinco postos de vacinação foram tomados por sorteio, como locais de coleta de dados, estimandose que em cada unidade seriam atendidas entre $100 \mathrm{a}$ 120 crianças.

Seis pessoas devidamente treinadas foram alocadas em cada unidade de vacinação, responsabilizando-se pela coleta de dados: preenchimento dos questionários (com perguntas fechadas e informações oriundas dos cartões de vacinação), medidas antropométricas, exame físico e coleta de amostra de sangue, além de um supervisor, respondendo pela coordenação local das operações.

As medidas de peso e altura foram obtidas segundo as recomendações técnicas da Organización Mundial de la Salud (OMS), 8 adotando-se, como referência o padrão do National Center of Health Statistics (NCHS), classificando-se as situações encontradas em escore-z (para crianças) ou índice de massa corporal (para adultos), segundo os critérios recomendados pela World Health Organization (WHO). 9 As determinações de hemoglobina foram realizadas através do método direto e instantâneo, utilizando-se o aparelho Hemocue, adotando-se o critério da OMS para a definição de anemia em mães e crianças. 10 Devido à disponibilidade de somente 3 Hemocues, a hemoglobina foi aferida em apenas 3 postos de vacinação.

Do questionário aplicado às mães, foram obtidos os dados referentes à idade, alfabetização, frequiência ao pré-natal e amamentação do último filho, assim como as informações anamnéticas aplicáveis às crianças, como queixas respiratórias, ocorrência de febre e diarréia e a participação em programa de recuperação nutricional.

Para fins de validação externa foram tomados como referência, os resultados da II Pesquisa Estadual de Saúde e Nutrição, 11 Pernambuco, realizada um ano e seis meses antes da presente pesquisa. As análises comparativas acham-se apresentadas na discussão do artigo.

\section{Aspectos éticos}

As mães foram informadas de que a participação no estudo seria voluntária, assegurando-se, de antemão, o anonimato da informação, explicitando-se que os resultados seriam utilizados para o conhecimento da situação de saúde da população e das condições de prestação de serviços e, ainda, que as informações poderiam vir a ser divulgadas em reuniões e revistas técnico-científicas. Os casos encontrados de anemia foram devidamente tratados, orientando-se, ademais, às mães sobre escolhas alimentares apropriadas para a correção ou prevenção da deficiência de ferro.

O protocolo da investigação foi submetido e aprovado pelo Comitê de Ética em Pesquisa do Departamento de Nutrição da Universidade Federal de Pernambuco.

\section{Resultados}

A pesquisa foi realizada numa amostra de 417 mães e 579 crianças, observando-se assim, que cada mãe levou ao posto de vacinação, em média, 1,4 crianças. Em sua grande maioria $(80,3 \%)$ as mães eram alfabetizadas, sendo capazes de escrever e ler um bilhete.

\section{Vacinação}

Quase a totalidade das mães $(96,7 \%)$ trouxe o "cartão da criança", instrumento onde estavam anotadas as aplicações de vacinas, a ocorrência de doenças, os registros gráficos do crescimento pôndero-estatural e do desenvolvimento psicomotor.

Na Tabela 2 estão sumarizados os resultados da vacinação, segundo registro no "cartão da criança". Entre as crianças com a idade de seis a 12 meses observou-se que apenas duas crianças $(7,4 \%)$ não tinham completado o esquema vacinal recomendado com a Sabin e a DPT, tendo recebido, portanto, menos de três doses.

A vacina contra tuberculose estava registrada em $98,0 \%$ dos cartões de vacinação, verificando-se através da inspeção a ocorrência de $95,2 \%$ de cicatrizes vacinais no braço direito das crianças. 
Tamanho da amostra e média aritmética do número de doses de vacina por faixa etária de crianças de 0 a 60 meses. Ribeirão, Pernambuco, 1998.

\begin{tabular}{|c|c|c|c|c|c|c|}
\hline \multirow{2}{*}{$\begin{array}{l}\text { Faixa etária } \\
\text { (meses) }\end{array}$} & \multicolumn{2}{|c|}{ Sabin } & \multicolumn{2}{|c|}{ DPT } & \multicolumn{2}{|c|}{ Sarampo } \\
\hline & Amostra & Média & Amostra & Média & Amostra & Média \\
\hline $0--6$ & 50 & 2,4 & 50 & 1,7 & 43 & 1,6 \\
\hline $7--12$ & 52 & 4,3 & 52 & 2,8 & 46 & 0,9 \\
\hline $13--24$ & 125 & 5,9 & 125 & 3,6 & 125 & 2,2 \\
\hline $25--48$ & 220 & 8,5 & 219 & 3,9 & 220 & 3,2 \\
\hline $49--60$ & 112 & 10,2 & 111 & 3,9 & 111 & 3,4 \\
\hline Total & 559 & 7,4 & 557 & 3,6 & 545 & 2,7 \\
\hline
\end{tabular}

DPT = Vacina contra Difteria, Coqueluche e Tétano.

\section{Morbidade}

Definiu-se, neste estudo, que se uma criança apresentasse concomitantemente queixa de dois dos três sintomas respiratórios pesquisados (tosse, cansaço, obstrução nasal) e ainda febre, nos últimos sete dias, seria considerada como um caso de infecção respi- ratória aguda. Por esse critério, observou-se que 104 crianças $(18,0 \%)$ apresentaram IRA nos últimos sete dias (Tabela 3). Nesta tabela se descreve, ainda, a ocorrência de diarréia nos últimos 15 dias, observando-se que a frequiência de casos aumenta progressivamente até os 24 meses de idade, declinando significativamente após esse limite de idade.

\section{Tabela 3}

Distribuição dos casos $(n)$ com sintomas positivos de diarréia e de infecção respiratória aguda por faixa etária de crianças de 0 a 60 meses. Ribeirão, Pernambuco, 1998.

\begin{tabular}{|c|c|c|c|c|c|c|}
\hline \multirow{2}{*}{$\begin{array}{l}\text { Faixa etária } \\
\text { (meses) }\end{array}$} & \multicolumn{3}{|c|}{ IRA } & \multicolumn{3}{|c|}{ Diarréia } \\
\hline & Amostra & $\mathrm{n}$ & $\%$ & Amostra & $\mathrm{n}$ & $\%$ \\
\hline $0--6$ & 50 & 8 & 16,0 & 49 & 6 & 12,2 \\
\hline $\begin{array}{lll}7 & -- & 12\end{array}$ & 52 & 11 & 21,2 & 50 & 11 & 22,0 \\
\hline $13--24$ & 127 & 22 & 17,3 & 123 & 34 & 27,6 \\
\hline $25--48$ & 229 & 35 & 15,3 & 221 & 21 & 9,5 \\
\hline $49--60$ & 120 & 28 & 23,3 & 120 & 11 & 9,2 \\
\hline Total & 579 & 104 & 18,0 & 563 & 83 & 14,7 \\
\hline
\end{tabular}

IRA = Infecção Respiratória Aguda 


\section{Estado nutricional}

Das 296 crianças que se submeteram ao exame de sangue, $112(37,8 \%)$ apresentaram níveis de hemoglobina menores que $11 \mathrm{~g} / \mathrm{dl}$, sendo, assim, anêmicas (Tabela 4). Na Tabela 5 estão resumidas as informações sobre os indicadores antropométricos, em termos de escores $z$. Observa-se que $11 \%$ das crianças apresentaram déficit estatural, enquanto os resultados mais favoráveis foram encontrados em relação ao índice peso/altura, com apenas $0,9 \%$ abaixo do valor crítico.

Observou-se que somente $0,9 \%$ das crianças estavam em programas de recuperação nutricional, se- gundo anotação no "cartão de vacinação" e que apenas em $12,2 \%$ e $2,9 \%$ estão anotadas informações sobre crescimento e desenvolvimento, respectivamente.

Quanto ao índice de massa corporal (IMC), das 412 mães estudadas constatou-se que existem duas vezes mais mulheres obesas que magras $(10,6 \%$ e $4,9 \%$ ) observando-se, também, um incremento expressivo da obesidade após os 30 anos de idade.

Os níveis de hemoglobina, evidenciaram que $16,2 \%$ das mães apresentaram valores menores que $12 \mathrm{~g} / \mathrm{dl}$, sendo consideradas anêmicas. Entre as mães de 15 a 19 anos encontrou-se a maior prevalência de anemia (Tabela 6).

Tabela 4

Distribuição de anemia e valores médios de hemoglobina (g/dl) por faixa etária, de crianças de 0 a 60 meses. Ribeirão, Pernambuco, 1998.

\begin{tabular}{|c|c|c|c|c|c|c|}
\hline \multirow{2}{*}{$\begin{array}{c}\text { Faixa etária } \\
\text { (meses) }\end{array}$} & \multirow[t]{2}{*}{ Amostra } & \multicolumn{2}{|c|}{ Anemia } & \multicolumn{3}{|c|}{ Hemoglobina } \\
\hline & & $n$ & $\%$ & $\bar{x}$ & \pm & $d p$ \\
\hline $0--6$ & 12 & 10 & 83,3 & 10,2 & \pm & 2,2 \\
\hline $7--12$ & 26 & 16 & 61,5 & 10,6 & \pm & 1,6 \\
\hline $13--24$ & 71 & 46 & 64,8 & 10,3 & \pm & 1,9 \\
\hline $25--48$ & 123 & 31 & 25,2 & 11,9 & \pm & 1,5 \\
\hline $49--60$ & 64 & 9 & 14,1 & 12,2 & \pm & 1,3 \\
\hline Total & 296 & 112 & 37,8 & 11,4 & \pm & 1,8 \\
\hline
\end{tabular}

Tabela 5

Distribuição, em escores $z$, dos indicadores antropométricos de crianças de 0 a 60 meses. Ribeirão, Pernambuco, 1998.

\begin{tabular}{|c|c|c|c|c|c|c|c|}
\hline \multirow{2}{*}{ Indicador } & \multirow{2}{*}{$\begin{array}{l}\text { Escore z } \\
\text { Amostra }\end{array}$} & \multicolumn{2}{|c|}{$<-2$} & \multicolumn{2}{|c|}{-2 a 2} & \multicolumn{2}{|c|}{$>2$} \\
\hline & & $\mathrm{n}$ & $\%$ & $n$ & $\%$ & $n$ & $\%$ \\
\hline Peso/Idade & 558 & 27 & 4,8 & 500 & 89,6 & 31 & 5,6 \\
\hline Altura/ldade & 555 & 11 & 11,0 & 485 & 87,4 & 9 & 1,6 \\
\hline Peso/Altura & 557 & 5 & 0,9 & 508 & 91,2 & 44 & 7,9 \\
\hline
\end{tabular}


Distribuição do Índice de Massa Corporal (IMC), valores médios de hemoglobina e prevalência da anemia ( $\mathrm{Hb}<12,0 \mathrm{~g} / \mathrm{dl})$, por faixa etária das mães de crianças de 0 a 60 meses. Ribeirão, Pernambuco, 1998.

\begin{tabular}{|c|c|c|c|c|c|c|c|c|c|c|c|c|c|}
\hline \multirow{3}{*}{$\begin{array}{l}\text { Faixa } \\
\text { Etária } \\
\text { (anos) }\end{array}$} & \multicolumn{9}{|c|}{ Índice de Massa Corporal (IMC - kg/m²) } & \multicolumn{4}{|c|}{ Hemoglobina (g/dl) } \\
\hline & \multirow{2}{*}{ Amostra } & \multicolumn{2}{|c|}{$<18,5$} & \multicolumn{2}{|c|}{$18,5-24,9$} & \multicolumn{2}{|c|}{$25-30$} & \multicolumn{2}{|c|}{$>30$} & \multirow{2}{*}{ Amostra } & \multirow{2}{*}{$\bar{X} \pm d p$} & \multicolumn{2}{|c|}{$\begin{array}{c}\text { Prevalência de } \\
\text { Anemia }\end{array}$} \\
\hline & & $\mathrm{n}$ & $\%$ & $\mathrm{n}$ & $\%$ & $\mathrm{n}$ & $\%$ & $\mathrm{n}$ & $\%$ & & & $\mathrm{n}$ & $\%$ \\
\hline $15-19$ & 60 & 5 & 8,3 & 40 & 66,7 & 9 & 15,0 & 6 & 10,0 & 30 & $13,3 \pm 1,6$ & 7 & 23,3 \\
\hline $20-29$ & 250 & 12 & 4,8 & 145 & 58,0 & 75 & 30,0 & 18 & 7,2 & 104 & $13,3 \pm 1,3$ & 14 & 13,5 \\
\hline $30-39$ & 83 & 3 & 3,8 & 36 & 46,2 & 26 & 33,3 & 18 & 16,7 & 27 & $12,9 \pm 1,7$ & 5 & 18,5 \\
\hline $40-49$ & 19 & 0 & 0,0 & 6 & 31,6 & 7 & 36,8 & 6 & 31,6 & 6 & $13,7 \pm 1,4$ & 1 & 16,7 \\
\hline Total & 412 & 20 & 4,9 & 227 & 55,8 & 117 & 28,7 & 48 & 10,6 & 167 & $13,2 \pm 1,4$ & 27 & 16,2 \\
\hline
\end{tabular}

\section{Amamentação e freqüência ao pré-natal}

As informações sobre amamentação do último filho menor de cinco anos mostraram que das 408 mães que responderam à questão, $343(84,1 \%)$ amamentaram seus filhos, sendo que dessas, 13 mães $(4,7 \%)$ já iniciaram a história alimentar dos recém-natos com a amamentação mista. A média e a mediana da amamentação exclusiva foram de 11,5 e 9,0 dias, res-pectivamente.

Quanto à frequiência às consultas nos exame prénatais, para a gravidez mais recente, $86,2 \%$ das mães afirmaram ter comparecido a pelo menos uma consulta, enquanto $50 \%$ das mães tinham se submetido a cinco ou mais consultas pré-natais.

\section{Discussão}

No Brasil já se conta com experiências isoladas utilizando a oportunidade do "dia nacional de vacinação" para efetuar estudos com enfoque epidemiológico sobre alguns problemas ou situações relacionadas com a saúde da criança. Assim, em 1989, Rissin 12 recorreu a um levantamento amostral de crianças que demandavam os postos especiais de vacinação, para realizar um estudo de validação dos dados produzidos em serviços públicos de saúde sobre a situação nutricional de menores de cinco anos. Mais recentemente Santos et al. 13 e Vasconcelos et al. 14 aproveitaram os dias nacionais de vacinação para avaliar a situação do aleitamento materno em duas capitais de estados do Brasil. Em duas oportunidades, 1996 e 1998, Brasil et al.15 estudaram amostras de, respectivamente, 3.750 e 3.857 crianças menores de doze meses, no município do Rio de Janeiro, para avaliar o perfil do aleitamento materno, recorrendo à demanda dos postos de vacinação.

O que agora se pretende é a utilização dessa oportunidade para a realização de estudos mais abrangentes, que possam responder, simultaneamente, a vários objetivos, seja sobre problemas, seja sobre cuidados de saúde prestados na área maternoinfantil, dentro das prioridades setoriais de âmbito nacional ou internacional. Essa abordagem contempla vários aspectos fundamentais que dificultam a realização de estudos epidemiológicos a partir de visitas domiciliares, como custos financeiros, logística e tempo gasto na coleta de dados, além de outros aspectos secundários.

A experiência piloto realizada na cidade de Ribeirão é bastante promissora. Assim, foi possível investigar uma amostra de 996 indivíduos (417 mães e 579 crianças) num período de oito horas de trabalho, a um custo operacional de $\mathrm{R} \$ 6.200,00$ (aproximadamente US $\$ 3.200,00$ ) o que corresponde a cerca de 3,5 dólares por pessoa. A relação custo/efetividade do método tornou-se, portanto, incomparavelmente melhor que a obtida por procedimentos convencionais dos estudos de base populacional, nos quais os custos, na experiência brasileira, sempre se situam acima de 15 dólares por unidade amostral. No caso de Pernambuco, por exemplo, a II Pesquisa Estadual de Saúde e Nutrição, 11 Pernambuco, realizada em 1997, cobrindo uma amostra de 1.190 mães e 1.970 crianças menores de cinco anos, foi orçada em US\$91.200,00, o que resultaria num custo de US $\$ 29,00$ por pessoa pesquisada. Há de se considerar que, neste caso, outras variáveis foram estudadas, como as condições socioeconômicas da família e o 
consumo alimentar numa subamostra, o que dificulta as inferências comparativas sobre custos ajustados para tornar a análise devidamente apropriada.

Questiona-se, em princípio, a possibilidade de resistências e dificuldades operacionais que potencialmente, afetariam o rendimento quali e quantitativo do inquérito. $\mathrm{Na}$ experiência aqui relatada a aceitação das equipes locais de vacinação foi muito boa, inclusive porque crianças e mães só eram examinadas após a aplicação das vacinas. O processamento local das amostras de sangue (centrifugação, separação do soro, rotulagem e congelamento do material biológico) tendo como apoio o laboratório de análises clínicas da Fundação Nacional de Saúde, não apresentou problemas, desde que dois veículos se revesavam, a cada 15 minutos, na operação de transporte das amostras.

A rapidez da realização da pesquisa constitui, certamente, a maior vantagem do método aqui proposto. Na II Pesquisa Estadual de Saúde e Nutrição, 11 já ressaltada, para uma amostra três vezes maior, a coleta de dados demandou um trabalho de campo de quatro meses, ou seja, 120 dias. Já a I Pesquisa Estadual de Saúde e Nutrição, Pernambuco, 16 realizada em 1991, representada por uma amostra de 835 crianças foi realizada, também, em 120 dias. No que se refere a coleta de dados de campo, a permanência média da equipe de pesquisadores em cada um dos 18 municípios foi de 5,2 dias. No segundo estudo, foram avaliadas, em média, 174 pessoas estudadas (109 crianças e 65 mães) por município, o que representou uma cobertura de 33,4 pessoas/dia por localidade estudada. Assim ajustada, a relação 996/33,4 representa um diferencial de quase 30 vezes em termos de economia de tempo, sempre com a observação de que nos diagnósticos do estado de Pernambuco foram utilizados instrumentos de coleta de dados bem mais complexos que os usados no estudo piloto de Ribeirão.

No aspecto logístico, as vantagens são evidentes, visto que as operações de transporte, comunicação, hospedagem, provisão de material, monitoramento e supervisão são bem mais simplificadas num estudo de um dia do que num trabalho prolongado por vários meses.

No que se refere à validação dos resultados, o fato de que se tenha cumprido o plano amostral dentro dos parâmetros estimados para cada variável de interesse (com exceção da hemoglobina, por limitação na quantidade de aparelhos), estudando-se 579 crianças residentes na área urbana da cidade, confere à amostra a desejada representatividade estatística.

Por outra parte, os resultados obtidos ocorreram dentro do esperado para populações urbanas do esta- do de Pernambuco, considerando-se os erros de estimativas amostrais pré-estabelecidos. A relação estatística mãe-filho menores de cinco anos (1,7 para o interior do Estado e 1,4 para a pesquisa simplificada) se explicaria seja por contingência da amostra, ou principalmente, pelo menor comparecimento de crianças menores de dois meses nos postos de vacinação. A ocorrência de $18 \%$ de IRA é plenamente comparável à prevalência de 22,3\% obtida para o interior urbano do Estado. A frequiência de diarréia nas duas últimas semanas diverge um pouco: $22,7 \%$ em crianças estudadas no domicílio versus $14,7 \%$ na amostra entrevistada no dia da vacinação. Isto poderia refletir o caráter sazonal das diarréias ou a relutância eventual de algumas mães em vacinar seus filhos na vigência de um processo diarréico.

Nas áreas urbanas do interior de Pernambuco, a prevalência da anemia, em menores de cinco anos, em 1997 foi de 40,6\%,11 equivalendo-se, portanto, à frequiência de $37,8 \%$ detectada na aplicação do modelo simplificado de diagnóstico na cidade de Ribeirão. Foram também semelhantes as prevalências de déficit estatural e ponderal ( $<-2$ escores $z)$ nos dois estudos: $12,1 \%$ versus $11,0 \%$ para o primeiro indicador e $4,9 \%$ versus $4,8 \%$ para o segundo. 11 Praticamente se eqüivaleram, também, as informações sobre o histórico da amamentação (freqüência e duração) e os dados sobre cobertura vacinal.

Em relação às mães, nos três aspectos considerados (assistência pré-natal em relação ao último filho, índice de massa corporal e níveis de hemoglobina), os resultados também foram comparáveis. Por outro lado, $86,2 \%$ das mães relataram ter frequientado serviços pré-natais, enquanto nas cidades de Pernambuco isso ocorreria com $87,1 \%$ das entrevistadas. 14 Os valores do IMC desenham o mesmo perfil encontrado em mulheres no meio urbano do estado e do Brasil.11,18

Mesmo com a observação de que os exames de hemoglobina realizados em Ribeirão não foram representativos (isto é, não alcançaram o tamanho amostral estimado), deve-se ressaltar que a freqüência de anemia, 16,2\%, não discrepa significativamente da prevalência estimada para mulheres do interior urbano do Estado: 19,9\%.11

Diante dos resultados, quais as perspectivas de desenvolvimento e aplicação do modelo?

A primeira questão, de ordem conceitual, consiste em referenciar que, sendo um modelo simplificado, não se poderia ampliar consideralvemente seu espectro de objetivos (e, portanto, de variáveis). É possível que o protótipo experimentado em Ribeirão tenha se aproximado do limite de compreensão de 
um instrumento consistente e ágil de coleta de dados de campo. Esse limite, pelos dados da experiência, poderia cobrir 10 a 12 objetivos, na dependência do objeto de estudo e do nível de detalhamento que se deseja alcançar. A avaliação do desenvolvimento de crianças menores de cinco anos poderia ser trabalhada pelo método aqui proposto. Circunstancialmente, os resultados da experiência de Ribeirão sobre o gráfico de desenvolvimento, que devem ser publicados num artigo em separado, não possibilitam, aqui, uma análise documentada sobre esse quesito, além da notificação de que sua abordagem se torna plenamente possível. Também se tornaria factível, evidentemente com novos procedimentos, incluir levantamentos do tipo screening sobre níveis séricos de retinol (vitamina A) em crianças, glicemia e lipidograma em mães (exames que implicam em jejum de 10 a 12 horas), testes sorológicos para doença de Chagas, sífilis e outras enfermidades.

Evidentemente o modelo não é inflexível, sendo vastíssimo o campo de variações que pode assumir. Pode-se conceber, por exemplo, estudos com vinte ou mais objetivos, desdobrados em dois ou mais sets de coleta de dados (dois momentos sucessivos de dias nacionais de vacinação, ou se fazer substituições ou permutas de objetivos e das variáveis que devem representá-las. São inúmeras, portanto, as perspectivas que podem ser consideradas para diferentes fins.

Embora testado em população urbana e em âmbito municipal, o modelo pode ser difundido para o meio rural e para um alcance espacial mais abrangente, incluindo estado ou região geográfica. Na realidade, um posto de vacinação comporta-se como um conglomerado (cluster), que atende pelo princípio da proximidade física ou facilidades de acesso, a um espaço e população relativamente bem delimitados. A coleta de dados simultaneamente em várias unidades de vacinação, com um plano amostral bem desenhado, poderia atender satisfatoriamente a essa perspectiva.

Um aspecto crucial, sem dúvida, é a necessidade de supervisão contínua e atenta de múltiplas equipes, numa operação intensiva de coleta de várias informações simultâneas. Esta é uma referência fundamental para a replicação da estratégia em futuros levantamentos.

No Brasil, o Centro Nacional de Epidemiologia (CENEPI) está estimulando o desenvolvimento do modelo, já dispondo, inclusive, de uma experiência aplicada em 13 localidades de 12 estados do país, replicando, com as devidas modificações, o protótipo experimental testado na cidade de Ribeirão.

\section{Referências}

1. UNICEF. Fundo das Nações Unidas para a Infância. Cuidados primários de saúde - Alma-Ata, 1978. Brasília, DF UNICEF; 1979.

2. Declaração mundial sobre a sobrevivência, a proteção e o desenvolvimento da criança e plano de ação para a implementação da Declaração mundial sobre a sobrevivência, a proteção e o desenvolvimento da criança nos anos 90. In: Encontro Mundial de Cúpula pela Criança; 1990 Set 28 29; New York, NY, Estados Unidos. Brasília, DF: Fundo das Nações Unidas para a Infância (UNICEF); 1990.

3. Informe final de la Conferencia Internacional sobre Nutricion.; 1992 Dec; Roma, Itália. Roma: Organización de las Naciones Unidas para la Agricultura y la Alimentacion (FAO), e Organización Mundial de la Salud (OMS); 1992

4. Batista Filho M. Saúde e nutrição. In: Rouquayrol MZ. Epidemiologia e saúde. 4 ed. Rio de Janeiro: Medsi; 1994.

5. Informe final de la Conferencia Mundial de los Alimentos; 1974 Nov. 5-6;. Roma, Itália. Roma: Organización de las Naciones Unidas para la Agricultura y la Alimentacion (FAO); 1974.
6. FAO. Organización de las Naciones Unidas para la Agricultura y la Alimentación. Sistema latinoamericano de monitereo alimentario y nutricional (SILMAN): estructura, organización y funcionamento. Santiago do Chile: FAO; 1997.

7. Pernambuco. FIDEM. Fundação de Desenvolvimento Municipal. Informações municipais. Recife: FIDEM; 1999.

8. OMS. Organización Mundial de la Salud. Medicion del cambio del estado nutricional: directrices para evaluar el efecto nutricional de programas de alimentación suplementaria destinados a grupos vulnerables. Ginebra: OMS; 1983.

9. WHO. World Health Organization. Physical status: the use and interpretation of antropometry: report of a WHO Expert Committee. Geneva: WHO; 1995. (Technical Report Series 854).

10. OMS. Organización Mundial de la Salud. Anemias nutricionais: informe de un. grupo de expertos de la OMS Ginebra: OMS; 1972. (Informes Tecnicos, 503).

11. Veras AACA, coordenadora. II Pesquisa Estadual de Saúde e Nutrição: saúde, nutrição, alimentação e condições so- 
cioeconômicas no estado de Pernambuco. Recife: Instituto Nacional de Alimentação e Nutrição do Ministério da Saúde (INAM/MS), Instituto Materno Infantil de Pernambuco (IMIP), Departamento de Nutrição da Universidade Federal de Pernambuco (DP/UFPE), Secretaria de Saúde do Estado de Pernambuco (SES/PE); 1998.

12. Rissin A. O SISVAM na campanha de vacinação. Bol SIS VAM 1989; 1: 11-14.

13. Santos EKA, Venâncio SI, Kitoko P, Réa MF, Monteiro CA. Diagnóstico rápido de práticas de alimentação infantil em campanhas de vacinação: a experiência de Florianópolis, SC, em 1997. In: Resumos do IV Congresso Brasileiro de Epidemiologia; 1998 ago 1-5; Rio de Janeiro, RJ. Rio de Janeiro: Associação Brasileira de Pós-Graduação em Saúde Coletiva (ABRASCO); 1998.

14. Vasconcelos ACCP, Venâncio SI, Kitoko P, Réa MF, Monteiro CA. Diagnóstico rápido de práticas de alimentação infantil em campanhas de vacinação: a experiência de João Pessoa, PB, em 1997. In: Resumos do IV Congresso Brasileiro de Epidemiologia; 1998 ago 1-5; Rio de Janeiro, RJ. Rio de Janeiro: Associação Brasileira de Pós-Graduação em Saúde Coletiva (ABRASCO); 1998.
15. Brasil CLP, Tavares EL, Castro IRR, Gomes MAM. Situação da alimentação materna no município do Rio de Janeiro, 1996 e 1998. In: I Encontro de Atualização em Nutrição Materno-Infantil; 1999 set. 22-29; Rio de Janeiro. Rio de Janeiro: Nestlé; 1998. (Serie Nestlé nutrição infantil)

16. UNICEF. Fundo das Nações Unidas para a Infância. Crianças e adolescentes em Pernambuco: saúde, educação e trabalho. Recife: UNICEF; 1992.

17. Lins e Silva SL. Cobertura e alguns aspectos qualitativos da assistência pré-natal no estado de Pernambuco [dissertação mestrado]. Recife: Instituto Materno Infantil de Pernambuco, IMIP; 2000.

18. Monteiro CA, Benício MHD, Freitas, ICM. Melhoria de indicadores de saúde associados à pobreza no Brasil nos anos 90: descrição, causas e impactos sobre desigualdades regionais. São Paulo: Núcleo de Pesquisas em Nutrição e Saúde da Universidade de São Paulo (NUPENS/USP); 1997. 